\title{
On the symmetries of a Rikitake type system
}

\author{
Cristian Lăzureanu and Tudor Bînzar \\ Department of Mathematics, "Politehnica" University of Timişoara \\ Piaţa Victoriei nr. 2, 300006 Timişoara, România \\ E-mail: cristian.lazureanu@upt.ro;tudor.binzar@upt.ro
}

\begin{abstract}
A symplectic realization and some symmetries of a Rikitake type system are presented. 1
\end{abstract}

Keywords: Rikitake system, symmetries, Hamiltonian dynamics.

\section{Introduction}

The Rikitake two-disk dynamo system is a mechanical model used to study the reversals of the Earth's magnetic field $(9])$.

This system has been widely investigated from different points of view. In a particular case, continuous symmetries were given by W.H.Steeb ([10]).

In this paper, considering another particular case of Rikitake system, namely

$$
\left\{\begin{array}{l}
\dot{x}=y z+\beta y \\
\dot{y}=x z-\beta x \\
\dot{z}=-x y
\end{array}\right.
$$

where $\beta \in \mathbf{R}$, some symmetries are given.

A similar study for Maxwell-Bloch equations was presented by P.A.Damianou and P.G.Paschali in 3 .

Theoretical details about symmetries of differential equations can be found in [1], [8], 4], [5], [2].

For our purposes, a Hamilton-Poisson realization and a symplectic realization of system (1.1) are required.

Symmetry and Hamiltonian systems are related in the topic "order in chaos". Physical systems often exhibit "order" simultaneous with symmetries. When the symmetry of a system is broken, Hamiltonian structures can be useful in detecting chaos 7 .

\section{Bi-Hamiltonian structure and symmetries}

In this section, we consider system (1.1) with $\beta=0$, i.e.

$$
\left\{\begin{array}{l}
\dot{x}=y z \\
\dot{y}=x z \\
\dot{z}=-x y
\end{array}\right.
$$

A bi-Hamiltonian structure and some symmetries of system (2.1) are presented.

Let us considering the three-dimensional Lie group of rigid motions of the Minkowski plane,

$$
E(1,1)=\left\{A \in G L(3, \mathbf{R}) \mid A=\left[\begin{array}{ccc}
1 & 0 & 0 \\
v_{1} & \cosh \theta & \sinh \theta \\
v_{2} & \sinh \theta & \cosh \theta
\end{array}\right], v_{1}, v_{2}, \theta \in \mathbf{R}\right\} .
$$

\footnotetext{
${ }^{1}$ COMPTES RENDUS MATHEMATIQUE Vol. 350, Issue: 9-10 (2012) Pages: 529-533; DOI: 10.1016/j.crma.2012.04.016
} 
The corresponding Lie algebra of $E(1,1)$ is

$$
e(1,1)=\left\{X \in g l(3, \mathbf{R}) \mid X=\left[\begin{array}{ccc}
0 & 0 & 0 \\
a & 0 & c \\
b & c & 0
\end{array}\right], a, b, c \in \mathbf{R}\right\} .
$$

Note that, as a real vector space, $e(1,1)$ is generated by the base $B_{e(1,1)}=\left\{E_{1}, E_{2}, E_{3}\right\}$, where

$$
E_{1}=\left[\begin{array}{rrr}
0 & 0 & 0 \\
-\frac{1}{2} & 0 & 0 \\
0 & 0 & 0
\end{array}\right], \quad E_{2}=\left[\begin{array}{rrr}
0 & 0 & 0 \\
0 & 0 & 0 \\
-\frac{1}{2} & 0 & 0
\end{array}\right], \quad E_{3}=\left[\begin{array}{rrr}
0 & 0 & 0 \\
0 & 0 & -\frac{1}{2} \\
0 & -\frac{1}{2} & 0
\end{array}\right]
$$

The following bracket relations $\left[E_{1}, E_{2}\right]=0,\left[E_{1}, E_{3}\right]=\frac{1}{2} E_{2},\left[E_{2}, E_{3}\right]=\frac{1}{2} E_{1}$, hold. by

On the dual space $e(1,1)^{*} \simeq \mathbf{R}^{3}$, the Lie-Poisson structure is given in coordinates using matrix notation

$$
\pi_{1}(x, y, z)=\left[\begin{array}{ccc}
0 & 0 & \frac{1}{2} y \\
0 & 0 & \frac{1}{2} x \\
-\frac{1}{2} y & -\frac{1}{2} x & 0
\end{array}\right]
$$

Following [11, considering the Lie group $O(Q)=\left\{A \in G L(3, \mathbf{R}) \mid A^{t} Q A=Q\right\}$ generated by $Q:=$ $\operatorname{diag}(2,1,1) \in G L(3, \mathbf{R})$, the corresponding Lie algebra is $o(Q)=\left\{X \in g l(3, \mathbf{R}) \mid X^{t} Q+Q X=O_{3}\right\}$. As a real vector space $o(Q)$ is generated by the base $B_{o(Q)}=\left\{X_{1}, X_{2}, X_{3}\right\}$, where

$$
X_{1}=\left[\begin{array}{rrr}
0 & 1 & 0 \\
-2 & 0 & 0 \\
0 & 0 & 0
\end{array}\right], \quad X_{2}=\left[\begin{array}{rrr}
0 & 0 & 1 \\
0 & 0 & 0 \\
-2 & 0 & 0
\end{array}\right], \quad X_{3}=\left[\begin{array}{rrr}
0 & 0 & 0 \\
0 & 0 & 1 \\
0 & -1 & 0
\end{array}\right] \text {. }
$$

The following bracket relations $\left[X_{1}, X_{2}\right]=-2 X_{3},\left[X_{1}, X_{3}\right]=X_{2},\left[X_{2}, X_{3}\right]=-X_{1}$, hold.

On the dual space $o(Q)^{*} \simeq \mathbf{R}^{3}$, the Lie-Poisson structure is given in coordinates using matrix notation by

$$
\pi_{2}(x, y, z)=\left[\begin{array}{ccc}
0 & -2 z & y \\
2 z & 0 & -x \\
-y & x & 0
\end{array}\right]
$$

Taking the constants of motion $H_{1}=\frac{1}{4} x^{2}-\frac{1}{4} y^{2}$ and $H_{2}=\frac{1}{2} x^{2}+\frac{1}{2} y^{2}+z^{2}$ of system (2.1), the following relations

$$
\pi_{1} \cdot \nabla H_{2}=\pi_{2} \cdot \nabla H_{1}=\left(\begin{array}{c}
y z \\
x z \\
-x y
\end{array}\right)
$$

hold.

Thus, system (2.1) is a bi-Hamiltonian system. For $\pi_{1}$ bracket, $H_{2}$ is the Hamiltonian and $H_{1}$ is a Casimir. For $\pi_{2}$ bracket, $H_{2}$ is the Hamiltonian and $H_{2}$ is a Casimir.

We recall that for a system $\dot{x}=f(x)$, where $f: M \rightarrow T M$, and $M$ is a smooth manifold of finite dimension, a vector field $\mathbf{X}$ is called:

- a Lie-point symmetry if its first prolongation transforms solutions of the system into other solutions;

- a conformal symmetry if the Lie derivative along $\mathbf{X}$ satisfies $L_{\mathbf{X}} \pi=\lambda \pi$ and $L_{\mathbf{X}} H=\nu H$, for some scalars $\lambda, \nu$, where the Poisson tensor $\pi$ and the Hamiltonian $H$ give the Hamilton-Poisson realization of the system;

- a master symmetry if $\left[\left[\mathbf{X}, \mathbf{X}_{f}\right], \mathbf{X}_{f}\right]=0$, but $\left[\mathbf{X}, \mathbf{X}_{f}\right] \neq 0$, where $\mathbf{X}_{f}$ is the vector field defined by the system.

The next result one furnishes a Lie point symmetry of system (2.1) and a conformal symmetry. 
Proposition 2.1. The vector field

$$
\mathbf{X}=-t \frac{\partial}{\partial t}+x \frac{\partial}{\partial x}+y \frac{\partial}{\partial y}+z \frac{\partial}{\partial z}
$$

is a Lie point symmetry of system (2.1). Moreover, $\mathbf{X}$ is a conformal symmetry.

Proof. If the vector $\mathbf{v}=\tau(t, x, y, z) \frac{\partial}{\partial t}+A_{1}(t, x, y, z) \frac{\partial}{\partial x}+A_{2}(t, x, y, z) \frac{\partial}{\partial y}+A_{3}(t, x, y, z) \frac{\partial}{\partial z}$ is a Lie point symmetry, then its first prolongation

$$
p r^{(1)}(\mathbf{v})=\mathbf{v}+\left(\dot{A}_{1}-\dot{\tau} \dot{x}\right) \frac{\partial}{\partial \dot{x}}+\left(\dot{A}_{2}-\dot{\tau} \dot{y}\right) \frac{\partial}{\partial \dot{y}}+\left(\dot{A}_{3}-\dot{\tau} \dot{z}\right) \frac{\partial}{\partial \dot{z}}
$$

applied to our system implies

$$
\left\{\begin{array}{l}
\dot{A}_{1}-y z \dot{\tau}-z A_{2}-y A_{3}=0 \\
\dot{A}_{2}-x z \dot{\tau}-z A_{1}-x A_{3}=0 \\
\dot{A}_{3}+x y \dot{\tau}+y A_{1}+x A_{2}=0
\end{array} .\right.
$$

One solution of above system is the vector $\mathbf{X}$.

One can easily check that

$$
L_{\mathbf{X}} \pi_{1}=-\pi_{1}, \quad L_{\mathbf{X}} \pi_{2}=-\pi_{2}, \quad L_{\mathbf{X}} H_{1}=2 H_{1}, \quad L_{\mathbf{X}} H_{2}=2 H_{2},
$$

whence $\mathbf{X}$ is a conformal symmetry.

The following result provides a master symmetry of our considered system.

Proposition 2.2. The vector field

$$
\vec{X}=\left(k_{1} x+k_{2} y z\right) \frac{\partial}{\partial x}+\left(k_{1} y+k_{2} x z\right) \frac{\partial}{\partial y}+\left(k_{1} z-k_{2} x y\right) \frac{\partial}{\partial z}, \quad k_{1} \in \mathbf{R}^{*}, k_{2} \in \mathbf{R},
$$

is a master symmetry of system (2.1).

Proof. We denote by $\vec{V}$ the associated vector field of system (2.1), that is $\vec{V}=y z \frac{\partial}{\partial x}+x z \frac{\partial}{\partial y}-x y \frac{\partial}{\partial z}$. It follows that the following relations $[\vec{X}, \vec{V}]=k_{1} \vec{V},[[\vec{X}, \vec{V}], \vec{V}]=0$ hold.

Therefore $\vec{X}$ is a master symmetry of system (2.1).

\section{Symplectic realization and symmetries}

Let us consider system (1.1) in the case $\beta \neq 0$.

In this section a symplectic realization of system (1.1) is given. Using this fact, the symmetries of Newton's equations are studied.

In order to obtain a Hamilton-Poisson realization of system (1.1), we again consider the Lie algebra $e(1,1)$ having now the base $B_{e(1,1)}^{\beta}=\left\{E_{1}^{\beta}, E_{2}^{\beta}, E_{3}^{\beta}\right\}$, where

$$
E_{1}^{\beta}=\left[\begin{array}{ccc}
0 & 0 & 0 \\
-\frac{1}{2 \beta} & 0 & 0 \\
0 & 0 & 0
\end{array}\right], E_{2}^{\beta}=\left[\begin{array}{ccc}
0 & 0 & 0 \\
0 & 0 & 0 \\
-\frac{1}{2 \beta} & 0 & 0
\end{array}\right], \quad E_{3}^{\beta}=\left[\begin{array}{ccc}
0 & 0 & 0 \\
0 & 0 & -\frac{1}{2 \beta} \\
0 & -\frac{1}{2 \beta} & 0
\end{array}\right]
$$

with $\left[E_{1}^{\beta}, E_{2}^{\beta}\right]=0,\left[E_{1}^{\beta}, E_{3}^{\beta}\right]=\frac{1}{2 \beta} E_{2}^{\beta},\left[E_{2}^{\beta}, E_{3}^{\beta}\right]=\frac{1}{2 \beta} E_{1}^{\beta}$.

Following [6], it is easy to see that the bilinear map $\Theta: e(1,1) \times e(1,1) \rightarrow \mathbf{R}$ given by the matrix $\left(\Theta_{i j}\right)_{1 \leq i, j \leq 3}, \Theta_{12}=-\Theta_{21}=1$ and 0 otherwise, is a 2-cocycle on $e(1,1)$ and it is not a coboundary since $\Theta\left(E_{1}^{\beta}, E_{2}^{\beta}\right)=1 \neq 0=f\left(\left[E_{1}^{\beta}, E_{2}^{\beta}\right]\right)$, for every linear map $f, f: e(1,1) \rightarrow \mathbf{R}$. 
On the dual space $e(1,1)^{*} \simeq \mathbf{R}^{3}$, a modified Lie-Poisson structure is given in coordinates by

$$
\Pi^{\beta}=\left[\begin{array}{ccc}
0 & 0 & \frac{1}{2 \beta} y \\
0 & 0 & \frac{1}{2 \beta} x \\
-\frac{1}{2 \beta} y & -\frac{1}{2 \beta} x & 0
\end{array}\right]+\left[\begin{array}{rcc}
0 & 1 & 0 \\
-1 & 0 & 0 \\
0 & 0 & 0
\end{array}\right]=\left[\begin{array}{ccc}
0 & 1 & \frac{1}{2 \beta} y \\
-1 & 0 & \frac{1}{2 \beta} x \\
-\frac{1}{2 \beta} y & -\frac{1}{2 \beta} x & 0
\end{array}\right]
$$

The Hamiltonian $H_{\beta}$ is given by $H_{\beta}(x, y, z)=\frac{\beta}{2} x^{2}+\frac{\beta}{2} y^{2}+\beta z^{2}$, and moreover, the function $C_{\beta}$, $C_{\beta}(x, y, z)=\frac{1}{4 \beta} x^{2}-\frac{1}{4 \beta} y^{2}+z$, is a Casimir of our configuration.

The next theorem states that the system (1.1) can be regarded as a Hamiltonian mechanical system.

Theorem 3.1. The Hamilton-Poisson mechanical system $\left(\mathbf{R}^{3}, \Pi^{\beta}, H_{\beta}\right)$ has a full symplectic realization $\left(\mathbf{R}^{4}, \omega, H\right)$, where $\omega=d p_{1} \wedge d q_{1}+d p_{2} \wedge d q_{2}$ and

$$
H=\frac{1}{16 \beta} q_{1}^{4}+\frac{1}{16 \beta} p_{1}^{4}-\frac{1}{8 \beta} q_{1}^{2} p_{1}^{2}-\frac{1}{2} q_{1}^{2} p_{2}+\frac{1}{2} p_{1}^{2} p_{2}+\frac{\beta}{2} q_{1}^{2}+\frac{\beta}{2} p_{1}^{2}+\beta p_{2}^{2} .
$$

Proof. The corresponding Hamilton's equations are

$$
\left\{\begin{array}{l}
\dot{q}_{1}=\frac{1}{4 \beta} p_{1}^{3}-\frac{1}{4 \beta} q_{1}^{2} p_{1}+p_{1} p_{2}+\beta p_{1} \\
\dot{q}_{2}=-\frac{1}{2} q_{1}^{2}+\frac{1}{2} p_{1}^{2}+2 \beta p_{2} \\
\dot{p}_{1}=-\frac{1}{4 \beta} q_{1}^{3}+\frac{1}{4 \beta} q_{1} p_{1}^{2}+q_{1} p_{2}-\beta q_{1} \\
\dot{p}_{2}=0
\end{array}\right.
$$

We define the application $\varphi: \mathbf{R}^{4} \rightarrow \mathbf{R}^{3}$ by

$$
\varphi\left(q_{1}, q_{2}, p_{1}, p_{2}\right)=\left(q_{1}, p_{1},-\frac{1}{4 \beta} q_{1}^{2}+\frac{1}{4 \beta} p_{1}^{2}+p_{2}\right)=(x, y, z) .
$$

It follows that $\varphi$ is a surjective submersion, the equations (3.1) are mapped onto the equations (1.1), the canonical structure $\{., .\}_{\omega}$ is mapped onto the Poisson structure $\Pi^{\beta}$, as required.

We also remark that $H_{\beta}=H$ and $C_{\beta}=p_{2}$.

From Hamilton's equations (3.1) we obtain by differentiation, Newton's equations:

$$
\begin{gathered}
\ddot{q}_{2} \dot{q}_{2}+2 \beta^{2} \ddot{q}_{2}+4 \beta^{2} q_{1} \dot{q}_{1}=0 \\
2 \beta \ddot{q}_{1} \dot{q}_{2}+4 \beta^{3} \ddot{q}_{1}-2 \beta \dot{q}_{1} \ddot{q}_{2}-\frac{1}{2 \beta} q_{1} \dot{q}_{2}^{3}-\beta q_{1} \dot{q}_{2}^{2}+2 \beta^{3} q_{1} \dot{q}_{2}+4 \beta^{5} q_{1}=0
\end{gathered}
$$

These are also Lagrange's equations generated by the Lagrangian

$$
L=\frac{1}{4 \beta} \dot{q}_{2}^{2}-\frac{\beta}{2} q_{1}^{2}+\frac{1}{4 \beta} q_{1}^{2} \dot{q}_{2}+\frac{\beta \dot{q}_{1}^{2}}{\dot{q}_{2}+2 \beta^{2}} .
$$

A vector field

$$
\vec{v}=\xi\left(q_{1}, q_{2}, t\right) \frac{\partial}{\partial t}+\eta_{1}\left(q_{1}, q_{2}, t\right) \frac{\partial}{\partial q_{1}}+\eta_{2}\left(q_{1}, q_{2}, t\right) \frac{\partial}{\partial q_{2}}
$$

is a Lie Point symmetry for Newton's equations if the action of its second prolongation on Newton's equations vanishes. Thus, the following conditions are obtained:

$\left(\dot{q}_{2}+2 \beta^{2}\right) \ddot{\eta}_{2}-\dot{q}_{2}\left(\dot{q}_{2}+2 \beta^{2}\right) \ddot{\xi}+4 \beta^{2} q_{1} \dot{\eta}_{1}+\ddot{q}_{2} \dot{\eta}_{2}-\dot{\xi}\left(4 \beta^{2} q_{1} \dot{q}_{1}+3 \ddot{q}_{2} \dot{q}_{2}+4 \beta^{2} \ddot{q}_{2}\right)+4 \beta^{2} \dot{q}_{1} \eta_{1}=0$

$2 \beta\left(\dot{q}_{2}+2 \beta^{2}\right) \ddot{\eta}_{1}-2 \beta \dot{q}_{1} \ddot{\eta}_{2}-4 \beta^{3} \dot{q}_{1} \ddot{\xi}-2 \beta \ddot{q}_{2} \dot{\eta}_{1}+\left(2 \beta \ddot{q}_{1}-\frac{3}{2 \beta} q_{1} \dot{q}_{2}^{2}-2 \beta q_{1} \dot{q}_{2}+2 \beta^{3} q_{1}\right) \dot{\eta}_{2}+\left(6 \beta \dot{q}_{1} \ddot{q}_{2}-6 \beta \ddot{q}_{1} \dot{q}_{2}-\right.$ $\left.8 \beta^{3} \ddot{q}_{1}+\frac{3}{2 \beta} q_{1} \dot{q}_{2}^{3}+2 \beta q_{1} \dot{q}_{2}^{2}-2 \beta^{3} q_{1} \dot{q}_{2}\right) \dot{\xi}+\left(-\frac{1}{2 \beta} \dot{q}_{2}^{3}-\beta \dot{q}_{2}^{2}+2 \beta^{3} \dot{q}_{2}+4 \beta^{5}\right) \eta_{1}=0$. 
The resulting equations obtained by expanding $\dot{\xi}, \ddot{\xi}, \dot{\eta}_{1}, \ddot{\eta}_{1}, \dot{\eta}_{2}, \ddot{\eta}_{2}$ must be satisfied identically in $t, q_{1}$, $q_{2}, \dot{q}_{1}, \dot{q}_{2}$, that are all independent. Doing standard manipulation, we get the overall result:

$$
\left\{\begin{array}{l}
\xi=c_{1} \\
\eta_{1}=0 \\
\eta_{2}=c_{2}
\end{array}\right.
$$

where $c_{1}, c_{2}$ are real constants.

For $c_{2}=0$, but $c_{1} \neq 0$, we have $\overrightarrow{v_{1}}=c_{1} \frac{\partial}{\partial t}$ that represents the time translation symmetry which generates the conservation of energy $H$.

For $c_{1}=0$, but $c_{2} \neq 0$, we have $\overrightarrow{v_{2}}=c_{2} \frac{\partial}{\partial q_{2}}$ that represents a translation in the cyclic $q_{2}$ direction which is related to the conservation of $p_{2}$.

Moreover, using the Lagrangian $L$ and Noether's theory we deduce that both $\overrightarrow{v_{1}}$ and $\overrightarrow{v_{2}}$ are variational symmetries since they satisfy the condition $\operatorname{pr}^{(1)} \vec{v}(L)+L \operatorname{div}(\xi)=0$.

\section{Acknowledgements}

We would like to thank the referees very much for their valuable comments and suggestions.

\section{References}

[1] G.W. Bluman, S. Kumei, Symmetries and Differential Equations, Appl.Math.Sci., No. 81, Springer Verlag, New York, 1989.

[2] P.A. Damianou, Multiple hamiltonian structures for Toda systems of type A-B-C, Regular and Chaotic Dynamics, V. 5, No. 1 (2000) 17-32.

[3] P.A. Damianou, P.G. Paschali, Symmetries of Maxwell-Bloch equations, J.Nonlinear Math.Phys., 2 N 3-4 (1995) 269-278.

[4] A.S. Fokas, B. Fuchssteiner, The Hierarchy of the Benjamin-Ono equations, Phys. Lett. 86 A (1981) $341-345$.

[5] B. Fuchssteiner, Mastersymmetries and higher order time-dependent symmetries and conserved densities of nonlinear evolution equations. Progr. Theor. Phys. 70 (1983) 1508-1522.

[6] P. Libermann, C.-M. Marle, Symplectic Geometry and Analytical Mechanics, D. Reidel, Dordrecht, 1987.

[7] J. Marsden, A. Weinstein, Coadjoint orbits, Vortices, and Clebsch variables for incompressible fluids, Physica 7D (1983) 305-323.

[8] P.J. Olver, Applications of Lie groups to Differential Equations, Springer Verlag, Neew York, 1986.

[9] T. Rikitake, Oscillations of a system of disk dynamos, Proc. Cambridge Philos. Soc., 54 (1958) 89-105.

[10] W.-H. Steeb, Continuous symmetries of the Lorenz model and the Rikitake two-disc dynamo system, J. Phys. A: Math. Gen., 15 (1982) 389-390.

[11] R.M. Tudoran, A. Aron, Ş. Nicoară, On a Hamiltonian Version of the Rikitake System, SIAM J. Applied Dynamical Systems, Vol. 8, No.1 (2009) 454-479. 